\title{
Hybrid Dysgenesis in Drosophila simulans Associated with a Rapid Invasion of the $P$-Element
}

\author{
Tom Hill, Christian Schlötterer, Andrea J. Betancourt* \\ Institut für Populationsgenetik, Vetmeduni Vienna, Austria \\ * andrea.betancourt@ vetmeduni.ac.at
}

\section{Gopenaccess}

Citation: Hill T, Schlötterer C, Betancourt AJ (2016) Hybrid Dysgenesis in Drosophila simulans Associated with a Rapid Invasion of the $P$-Element. PLoS Genet 12(3): e1005920. doi:10.1371/journal. pgen. 1005920

Editor: Harmit S. Malik, Fred Hutchinson Cancer Research Center, UNITED STATES

Received: October 26, 2015

Accepted: February 14, 2016

Published: March 16, 2016

Copyright: ๑ 2016 Hill et al. This is an open access article distributed under the terms of the Creative Commons Attribution License, which permits unrestricted use, distribution, and reproduction in any medium, provided the original author and source are credited.

Data Availability Statement: Sequence data are available from Genbank under Accession numbers PRJNA308281 and KU719478-KU719502.

Genotyping and cytotyping results are available from Dryad under doi:10.5061/dryad.1rq8f.

Funding: This work was funded by a grant P27048 from the Austrian Science Fund (FWF) to AJB. The funders had no role in study design, data collection and analysis, decision to publish, or preparation of the manuscript.

Competing Interests: The authors have declared that no competing interests exist.

\section{Abstract}

In a classic example of the invasion of a species by a selfish genetic element, the $P$-element was horizontally transferred from a distantly related species into Drosophila melanogaster. Despite causing 'hybrid dysgenesis', a syndrome of abnormal phenotypes that include sterility, the $P$-element spread globally in the course of a few decades in $D$. melanogaster. Until recently, its sister species, including $D$. simulans, remained $P$-element free. Here, we find a hybrid dysgenesis-like phenotype in the offspring of crosses between $D$. simulans strains collected in different years; a survey of 181 strains shows that around $20 \%$ of strains induce hybrid dysgenesis. Using genomic and transcriptomic data, we show that this dysgenesisinducing phenotype is associated with the invasion of the $P$-element. To characterize this invasion temporally and geographically, we survey $631 D$. simulans strains collected on three continents and over 27 years for the presence of the $P$-element. We find that the $D$. simulans $P$-element invasion occurred rapidly and nearly simultaneously in the regions surveyed, with strains containing $P$-elements being rare in 2006 and common by 2014. Importantly, as evidenced by their resistance to the hybrid dysgenesis phenotype, strains collected from the latter phase of this invasion have adapted to suppress the worst effects of the $P$-element.

\section{Author Summary}

Some genes perform necessary organismal functions, others hijack the cellular machinery to replicate themselves, potentially harming the host in the process. These 'selfish genes' can spread through genomes and species; as a result, eukaryotic genomes are typically saddled with large amounts of parasitic DNA. Here, we chronicle the surprisingly rapid global spread of a selfish transposable element through a close relative of the genetic model, Drosophila melanogaster. We see that, as it spreads, the transposable element is associated with damaging effects, including sterility, but that the flies quickly adapt to the negative consequences of the transposable element. 


\section{Introduction}

Discovered in the 1950's by Barbara McClintock [1], transposable elements (TEs) are the 'ultimate parasite' $[2,3]$. They are genetically simple, with autonomous elements consisting of as litthe as one protein coding gene, but remarkably successful, comprising much of the bulk of large eukaryotic genomes $[4,5]$. Those that persist do so by inserting copies of themselves into new genomic locations faster than they are eliminated. TEs can also be a source of beneficial mutations; particular TE insertions have been implicated in a number of adaptive phenotypes (reviewed in [6]), and some TE families now form essential components of genomes [7,8]. But most TE insertions are probably deleterious, as insertions can damage genes and may impose other fitness costs on their host [9-11]. In fact, the mutagenic properties of transposons have been harnessed by molecular geneticists to disrupt thousands of Drosophila genes (e.g., [12]).

In the short-term, transposable elements are vertically transmitted, and so can only cause limited damage to their hosts without extinguishing themselves. In the long-term, however, they are also horizontally transmitted [13-15], and, initially, may cause substantial damage to their hosts $[16,17]$. The best-studied example of a horizontally transferred element is that of the $P$-element, a DNA transposon. $P$-element was acquired by $D$. melanogaster by horizontal transfer from a distant relative, D. willistoni [18], and spread rapidly through the species, as evidenced by its absence from old laboratory stocks collected before the $P$-element was widespread [19-21].

In its native species, and in current $D$. melanogaster populations, the $P$-element is not associated with any gross defects $[18,22]$. But when males from strains collected after the invasion ('P strains') are crossed to females from old laboratory stocks ('M strains'), the F1 offspring suffer a number of abnormalities, including recombination in males, increased mutation rates, frequent sterility, and abnormally small (dysgenic) gonads [16,19,23-25]. These abnormalities, collectively called 'hybrid dysgenesis', appear to be due to DNA damage caused by actively transposing $P$-element in the germline $[17,22,26,27]$. In the reciprocal direction, the F1 offspring, though genetically identical, are normal. The reason is that these offspring are protected by piRNAs (PIWI interacting RNAs, a type of small RNA) homologous to the $P$-element, which are sequestered in the egg cytoplasm of P-type females. As these protective piRNAs target the $P$-element mRNA for degradation [28], their presence reduces $P$-element activity and the resulting DNA damage that lead to hybrid dysgenesis.

Since its initial discovery, $P$-element has been one of the most important tools in Drosophila genetics, providing the basis for genetic manipulations and mutagenesis (e.g., [12,29]). The Pelement had been thought to be absent from D. melanogaster's close relatives [30], but recent work has detected it in D. simulans, apparently horizontally acquired from D. melanogaster [31]. As the D. simulans elements recovered are identical in sequence to a type that segregates at low frequency in D. melanogaster, and do not include the most common D. melanogaster element, this acquisition is likely due to a single transfer event [31]. Here, we report a hybrid dysgenesis-like phenotype in crosses between strains from different $D$. simulans populations, and show this phenotype occurs in crosses between strains collected before and after a rapid invasion of the $P$-element.

\section{Results}

\section{Hybrid dysgenesis in $D$. simulans}

We assayed for a hybrid dysgenesis-like phenotype in D. simulans, using the absence of developed ovaries as an indicator (Fig 1 and S1 Fig, see also [24,32]). We considered a pair of strains to show hybrid dysgenesis when the F1 females from reciprocal crosses show a significant 

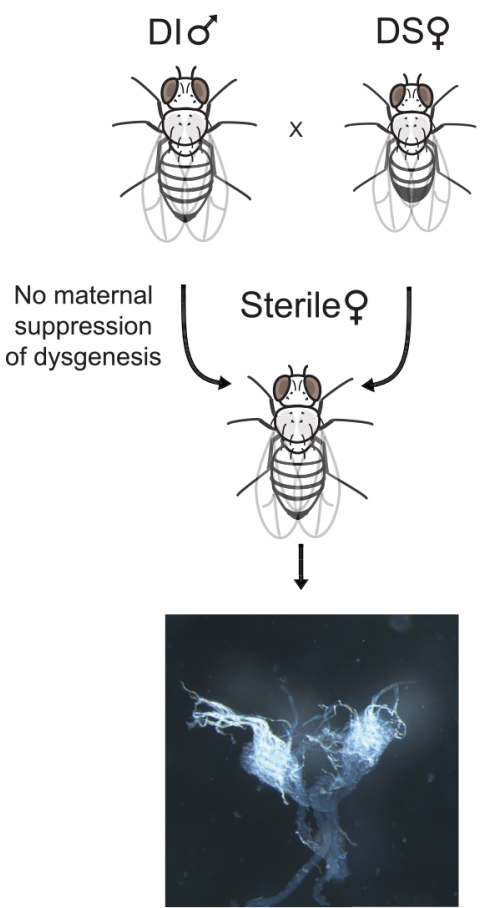
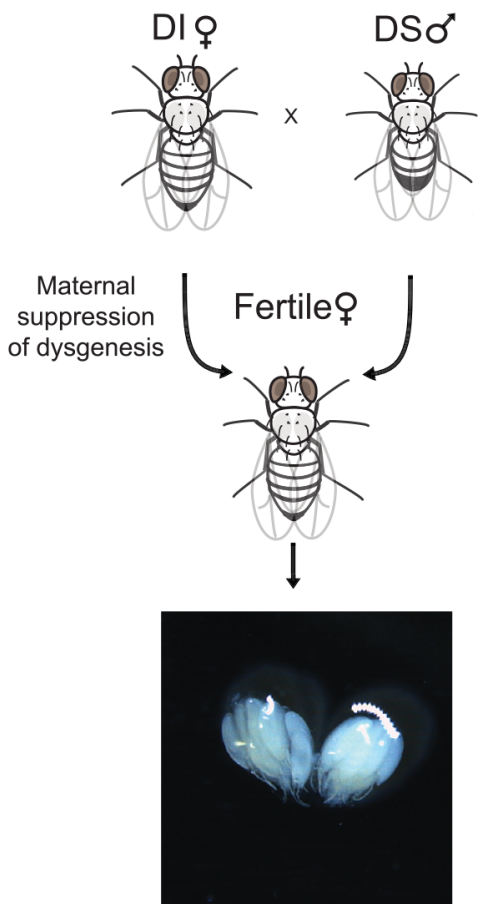

Fig 1. Hybrid dysgenesis in $\boldsymbol{D}$. simulans. Outcome of a reciprocal cross between $D$. simulans strains. In the non-dysgenic direction of the cross (left, FL31o $\times$ M2520'), most female offspring have normal ovaries, while in the reciprocal, non-dysgenic cross (right, FL31 $0^{7} \times$ M252\%), many female offspring suffer from malformed ovaries.

doi:10.1371/journal.pgen.1005920.g001

difference in the number of dysgenic ovaries [with significance defined as $p \leq 0.05$ in a Fisher's exact test (FET)]. Differences between F1 females from reciprocal crosses, which have identical nuclear genotypes, are likely due to different maternal contributions to the egg cytoplasm and implicate a cyto-nuclear incompatibility, as in the D. melanogaster hybrid dysgenesis system $[19,28]$.

For our initial survey, we used 22 strains collected in Georgia in 2009 (generously donated by P. Haddrill) and 10 strains collected in Madagascar in $2004 \& 2008$ (collected by B. Ballard and J. David; S1 Table). We paired strains from the two populations randomly, but ensured that each strain was involved in multiple crosses (at least 5 for the Madagascar strains and at least 2 for the Georgia strains). Among these crosses, 38.7\% (36 of 93) showed unidirectional gonadal dysgenesis in $\mathrm{F} 1$ females at $29^{\circ} \mathrm{C}$, a temperature which induces hybrid dysgenesis in $D$. melanogaster, but not at $25^{\circ} \mathrm{C}$ (S2 Fig). In addition to transposable elements, cyto-nuclear incompatibilities can also be caused by the intracellular bacteria Wolbachia. This is particularly likely here, as Georgia lines harbor a Wolbachia strain $[33,34]$ which appears to induce embryonic lethality in the offspring of crosses to Madagascar males. When we repeated the crosses after curing the strains of their Wolbachia infections, however, we found qualitatively similar results for infected and uninfected versions of the strains (S2 Fig, FET on data from the same cross performed with cured and uncured strains, $p>0.05$ for all).

Inspection of the data shows that dysgenic crosses almost always involved one of five Georgia lines as the paternal line (S2 Fig). Control crosses between these "dysgenesis-inducing" (DI) lines showed no significant unidirectional dysgenesis, nor did crosses of these DI lines to 12 of the other Georgia lines (a subset of crosses are shown in S2C and S2D Fig). In contrast, all 10 strains from Madagascar were "dysgenesis-susceptible" (DS) when crossed to DI lines; crosses 


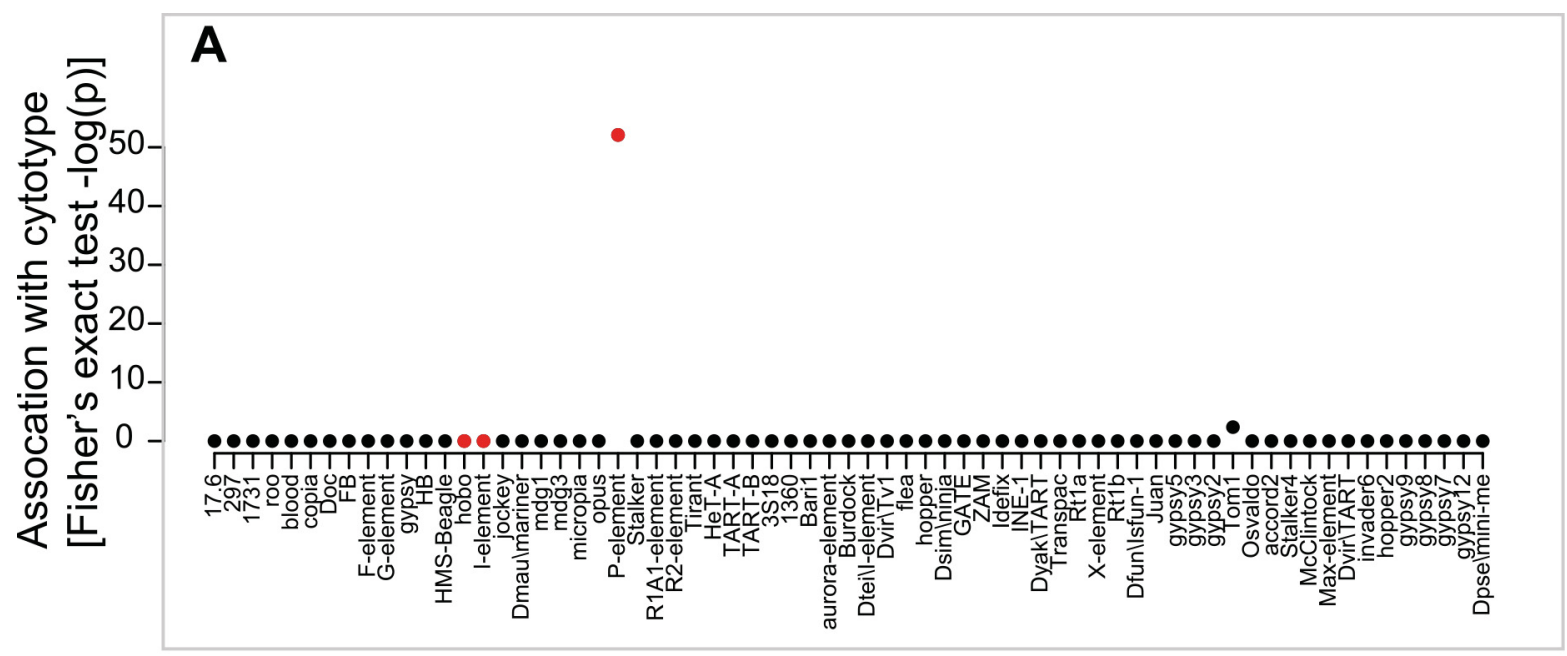

Transposable element family

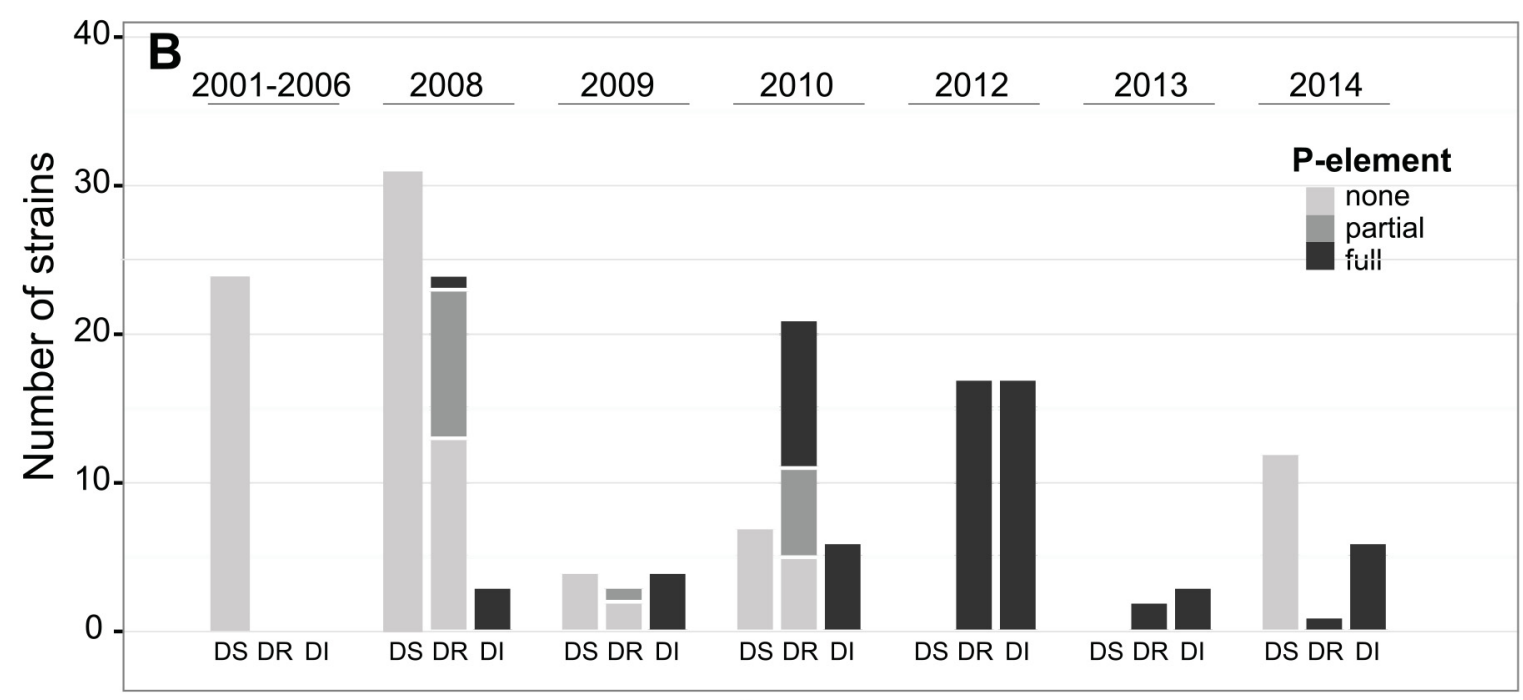

Cytotype

Fig 2. Cytotypes of lines assayed for dysgenesis. A) Plot showing the significance of association between the presence of different TE families in lines and their ability to cause hybrid dysgenesis $(-\log (\mathrm{p})$ from Fisher's exact tests). TEs that previously have been associated with hybrid dysgenesis $(P$-element, hobo, I-element) are highlighted in red. B) The cytotypes and $P$-element infection status of a worldwide sample of $D$. simulans lines is shown. Populations from the same year are grouped together by cytotype. Two strains showed amplification of all four exons individually, but not of full-length element.

doi:10.1371/journal.pgen.1005920.g002

between these lines also resulted in no hybrid dysgenesis (S2 Fig). The DI and DS classes are analogous to the P-type and M-type of D. melanogaster, respectively.

Using the Georgia and Madagascar strains with known DI and DS phenotypes, we unambiguously cytotyped 160 additional strains (cured of Wolbachia) from 23 populations sampled between 2002 and 2014 (Fig 2 and S3 Fig, S1 Table). We reciprocally crossed each strain to at least one known DI and DS strain, and roughly classified tested strains based on the results of the four crosses (see Materials and Methods). In addition to DI and DS types, we also observed a third type of strain, "dysgenesis-resistant" (DR), which produce no dysgenic offspring in either cross. These correspond to the $\mathrm{Q}$ types in D. melanogaster, which repress transposition of the $P$-element, but which contain few or no active elements $[25,35]$. In fact, the resistant strains probably encompass a range of intermediate cytotypes (i.e., corresponding to the M', Q 
and weak P-types in D. melanogaster [17,25,35-38]). It is likely that these strains contain copies of the dysgenesis-causing element, but in degraded form, at low copy number, or with weak expression. These copies may nevertheless be able to produce piRNAs suppressing the TE $[28,39]$.

Overall, $19.3 \%$ of strains were DI, $26.5 \%$ DR, and $54.2 \%$ DS. Fertility assays performed on a subset of these crosses show that F1 females from dysgenic crosses show higher rates of sterility and produce fewer offspring than their genetically identical sisters from reciprocal crosses (S4 Fig; Mann-Whitney U test, $p<0.05$ ).

The $D$. simulans hybrid dysgenesis phenotype is associated with the P-element. Given the similarity between transposable element-induced dysgenesis in D. melanogaster and the results from the D. simulans crosses, we suspected that the DI lines might have a dysgenesisinducing transposable element that is absent from the DS lines. Since the initial discovery of $P$ element, two other hybrid dysgenic systems have been discovered in D. melanogaster, the hobo-empty and inducer-reactor systems [40-42] and one in D. virilis, at least partly due to the Penelope element [43]. The existence of other dysgenesis-inducing TEs suggests that $P$-element might not be the cause of the hybrid dysgenesis seen here.

To identify TE families potentially associated with the dysgenesis-inducing agent, we used RNAseq data collected from pooled lines from a Florida population (originally collected in 2010, data from [31]), which shows both DI and DR cytotypes. We found 68 expressed TE families in these data. We investigated whether any of these elements appear to be associated with the observed hybrid dysgenesis, using three sources of evidence. First, we used genome sequence data from 12 single F1 females, produced by crossing males from the Florida population (4 DI strains, 6 DR strains \& 3 DS strains collected in Florida 2010) to females from a single Madagascar strain, M252 (a DS strain, collected in Madagascar in 2004 [44], which lacks $P$ elements). We examined these sequences for TE families present in the Florida lines, but absent from M252. Only one of the expressed TE families showed an appreciable level of coverage and differed between the F1 flies and the M252 line-the P-element (S2 Table and S5 Fig). With short-read sequence data, we could not unambiguously identify full-length insertions, but at least three of the sequenced strains showed coverage of the complete $P$-element reference sequence [31].

Second, we looked for an effect of $P$-element copy number on the level of dysgenesis, as strains with more $P$-element copies are likely to suffer from more DNA damage in the presence of active transposase than those with fewer copies (see [16]). As a proxy for copy number in the Florida lines, we used sequence coverage of the complete P-element reference in the genome sequence data from the 12 F1 Florida X M252 females. Consistent with expectations, the strength of dysgenesis increases with coverage of the $P$-element in crosses where the male parent is from Florida [with coverage of the $P$-element standardized by the average coverage of the euchromatic genome; S6 Fig; binomial generalized linear model (GLM); $z=6.49, p=8.49 \mathrm{x}$ $10^{-11}$. There is no relationship between dysgenesis and coverage for the reciprocal cross $(z=$ $-1.128, p=0.26$ ), or for coverage of other TEs in any cross ( $p>0.05$ for all, S2 Table).

Finally, we designed primers within the exons of the 68 expressed TEs (S2 Table), and tested for differences between previously cytotyped DI and DS strains using PCR. All but two of the elements with appreciable coverage were found to be either present in or absent from all strains, regardless of cytotype ( $P$-element and Tom1; S2 Table). Tom1 is not significantly associated with cytotype (present in 35 of 35 DI strains, and 71 of 78 DS strains, FET $p=0.097$ ), and there was no relationship between sequence coverage of Tom 1 and the strength of dysgenesis, as described above (S6 Fig; binomial GLM; $z=-0.551, p=0.581$ ). In contrast, the presence of $P$-elements is strongly associated with the DI cytotype, with products corresponding to $P$ element (a subset of which were verified by Sanger sequencing) amplified from all 35 DI 
strains. We were also able to amplify full-length $P$-element from 31 of these strains using standard PCR, and from the 4 remaining strains using RT-PCR-we were able to amplify all 4 exons of the $P$-element individually in all of the 35 strains. In contrast, no $P$-element could be amplified from any of the 98 DS strains (full-length element obtained using standard PCR in DI vs. DS strains; FET $p<2.2 \times 10^{-16}$ ). Using RT-PCR, we confirmed that full-length $P$-element is expressed in the 5 DI strains used in experimental crosses for cytotyping, and that no full-length transcript could be amplified from the DS strains used (S7A Fig).

Based on these three lines of evidence, we conclude that the $P$-element is the likely cause of the dysgenesis we see in D. simulans. In fact, the $P$-element is known to be active and cause dysgenesis in $D$. simulans when artificially introduced $[32,45]$. Consistent with a homology-based silencing mechanism, such as that mediated by piRNAs [28], maternal suppression of dysgenesis appears to be associated with the presence of the $P$-element in some form, either partial or full-length: we were able to amplify $P$-element sequence from all 48 DR strains (48 DR strains with $P$-element $v s .98$ empty DS strains; FET $p<2.2 \times 10^{-16}$ ). In 18 of the 48 DR strains, we found only partial copies of the $P$-element, consistent with the production of piRNAs from degraded copies of the transposon [46,47]. If, as in D. melanogaster, the maternal suppression of $P$-element sequence in a bi-directional piRNA cluster (one to which both sense and antisense piRNAs map) [46-48], the resistant strains might express both sense and anti-sense transcripts). We performed RT-PCR for each direction of the transcript separately on 10 strains (3 DI, 4 DR, 3 DS), and, as expected, found expression for both the sense and anti-sense RNA strands for DI and DR lines, but for neither direction in the DS lines (S7B Fig).

\section{The $P$-element invasion of $D$. simulans occurred rapidly on three continents}

We reasoned that, as in D. melanogaster, the DS and DI strains might show temporal and/or geographical differences $[20,21,49-51]$. In fact, the frequency of the DI and DR dysgenesis types increase over time (binomial GLM; Africa: $z=5.822, p=5.81 \times 10^{-9}$ ), and previous work shows differences in $P$-element content between a samples from 2010 and 2012 [31]. We tested a collection of 631 samples (isofemale lines, ethanol-preserved flies, and DNA extracted from wild flies) collected from 54 locations between 1984 and 2014 for the presence or absence of the $P$-element (S1 Table, Figs 3 and 4). To detect $P$-element, we used PCR, as before, with multiple sets of primers and repeated independent DNA extractions (see Materials and Methods). As before, the primers were used to amplify products that correspond to the full-length element and to each of the four exons. As for the cytotyped lines, we found strains with full-length copies, strains with only partial copies (in which at least one exon consistently failed to amplify), and strains with no successful amplification of any of the tested $P$-element fragments. The partial copies were usually missing internal exons, a common by-product of imperfect excision events in D. melanogaster [16,52]. Strains with full-length elements often also contained other, partial copies with similar internal deletions.

The fraction of strains containing the $P$-element increases over time on all continents surveyed. In collections from 2004 to 2009, strains free of the $P$-element predominate (Fig 3A). After 2009, most strains harbour some form of $P$-element, and many have full-length elements (Fig 3B). D. sechellia, a close relative of D. simulans with which it is known to hybridize [53,54], remains $P$-element free in our 2014 samples (kindly provided by D. Matute, Fig 3B). We find a significant association between when a strain was collected and whether it contains any $P$-element copies, regardless of the geographic region from which it was collected (Fig 4, binomial GLM with year, geographical region, and an interaction term fit to the $P$-element for each strain; year as $z=5.218 p=1.81 \times 10^{-7}$ ). 
A

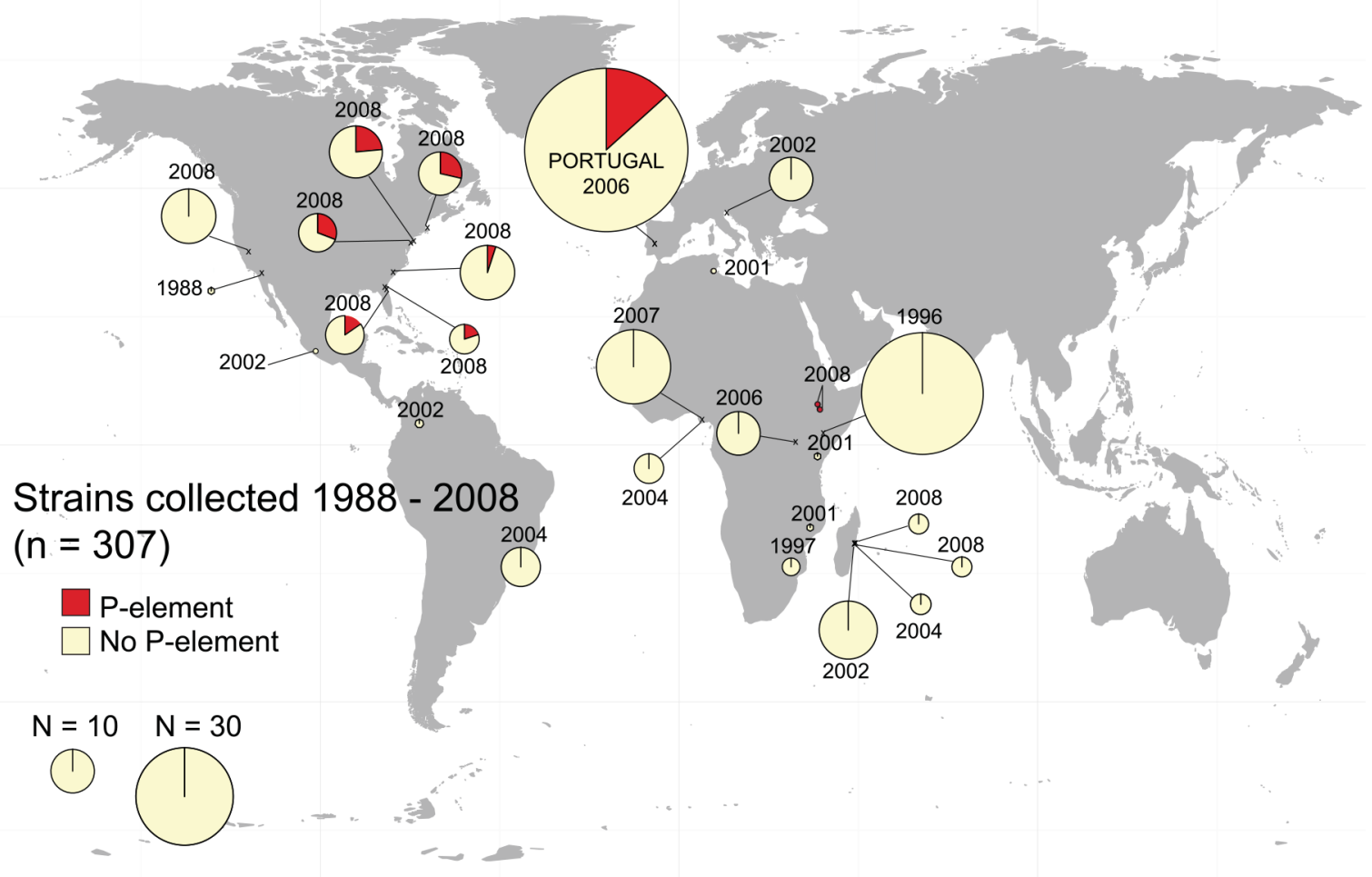

B

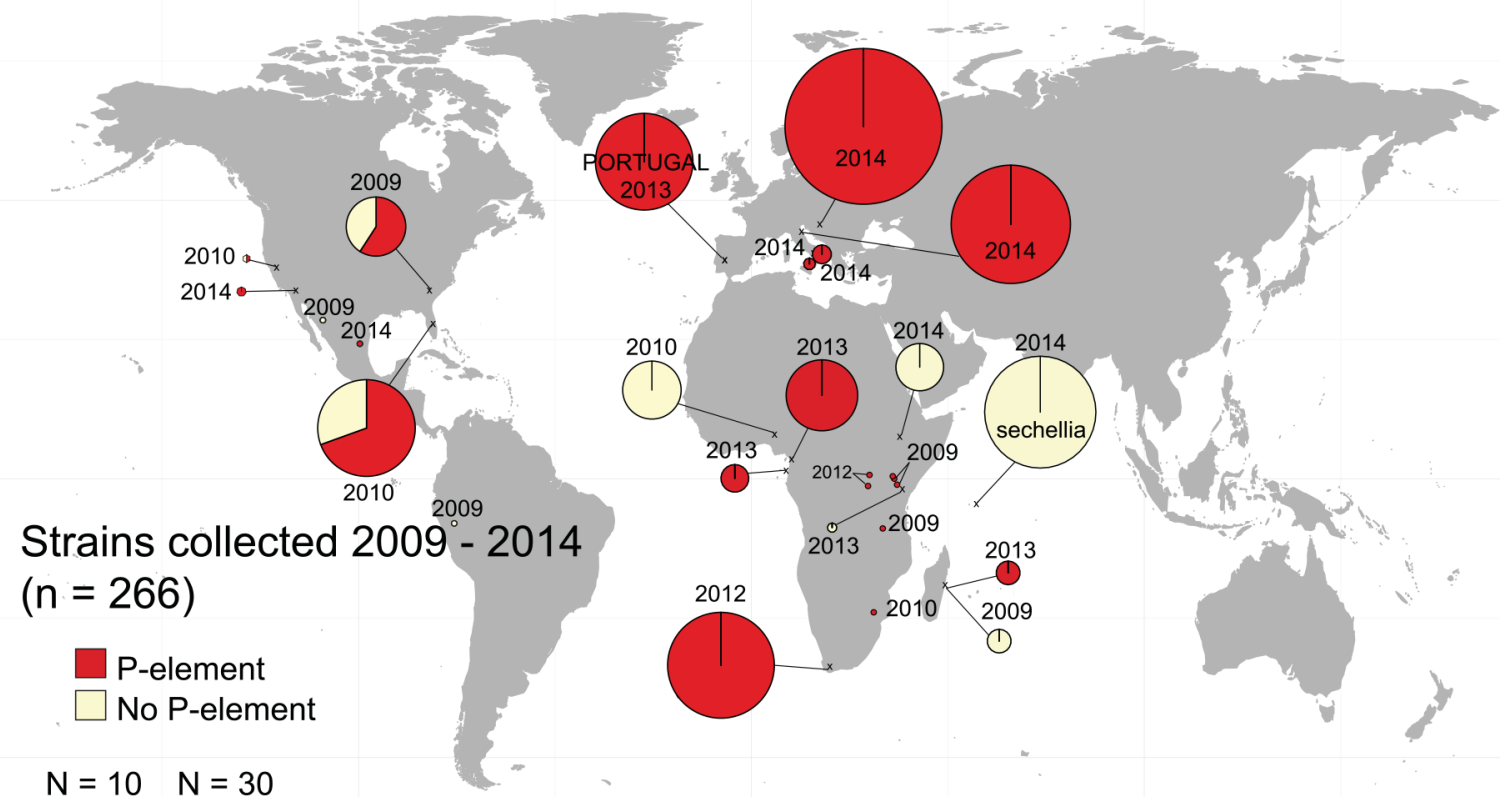

(1)

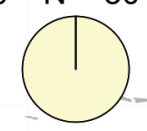


Fig 3. Partial and full $P$-element copies in $D$. simulans collected A) 1984-2008, and B) 2009-2014. Map shows the approximate location where strains were collected, and pie charts show the proportion of strains containing $P$-element (either full-length copies or individual exons could be amplified by PCR) and no copies (no $P$-element of any kind could be sampled). The area of the pie charts is proportional to the number of strains sampled (see Legend), except for single points, which indicate one strain.

doi:10.1371/journal.pgen.1005920.g003

By 2013, almost all sampled strains contained $P$-elements in some form (Fig 3). It is formally possible that this apparent spread is instead due to the loss of $P$-element in strains propagated for several years in the laboratory. This process could not have occurred, however, in the Portuguese samples, for which genomic DNA was extracted from directly wild flies, and these show a similar pattern (compare Portuguese samples from 2006 and 2013 in Fig 3). The rapidity of the spread makes it difficult to identify a likely origin point for the invasion, as the $P$-element appears to have invaded all three regions nearly simultaneously (Fig 4), with statistical differences between regions (S3 Table) apparently due to low prevalence of the $P$-element in a few late samples, rather than pointing to a geographic origin of the invasion.

\section{Discussion}

Here, we identified a hybrid dysgenesis-like system in D. simulans. Using next generation sequencing information and PCR, we find that the $P$-element, which has recently invaded $D$. simulans [31], is associated with the hybrid dysgenesis phenotype, and spread rapidly across Europe, Africa, and North America. In fact, compared to D. melanogaster, the spread of the $P$ element in $D$. simulans is surprisingly fast: In D. melanogaster, the first $P$-type fly was collected from the wild in 1954, and most wild strains were infected by 1974 [20]. We find the first evidence of the $P$-element in $D$. simulans samples from 2006, with $P$-elements appearing in nearly all lines sampled since 2013, seven years later (apart from one sample from Ethiopia; Fig 3, Fig 4, S1 Table).

There are a number of reasons the $P$-element invasion may have occurred more rapidly in $D$. simulans than in D. melanogaster. For one, $D$. simulans appears to enjoy lower rates of $P$-element induced gonadal dysgenesis than $D$. melanogaster; high rates of sterility are expected to hamper the spread of the $P$-element by reducing the fitness of its carriers. Direct comparisons between the species are problematic, as the severity of hybrid dysgenesis increases with copy

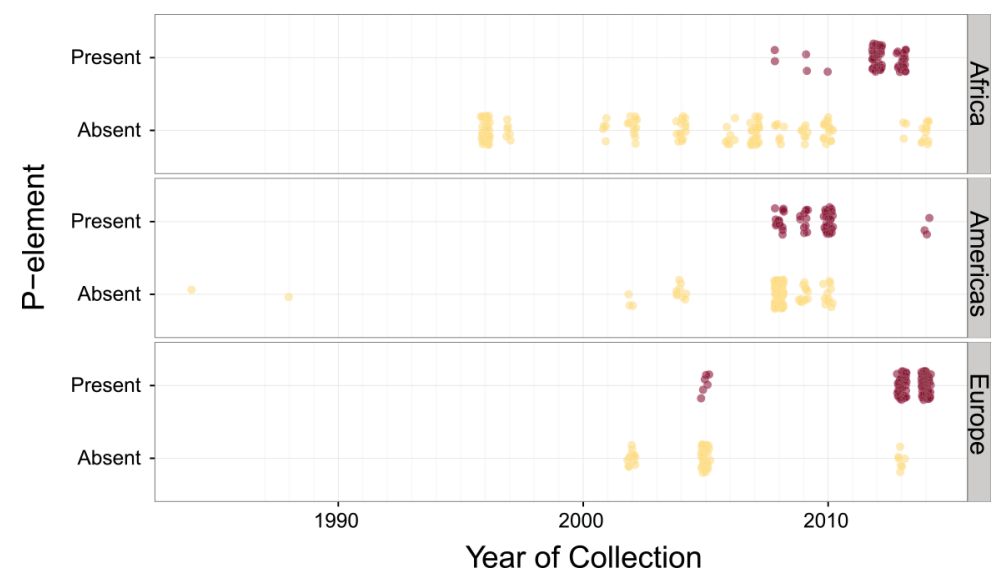

Fig 4. Time series of $P$-element in different forms in $D$. simulans. The plot shows strains with full (and partial) and no $P$-elements over time in Africa (top panel), North and South America (middle panel), and Europe (bottom panel) across time. Each point indicates a strain, with overlapping points jittered slightly. Data from the 1980's in the Americas are from Brookfield (1984) [30].

doi:10.1371/journal.pgen.1005920.g004 
number, which differs between the species ([19,24,26,31]; S2, S4 and S6 Figs). But in comparisons between artificially constructed $D$. melanogaster and $D$. simulans strains with comparable copy numbers, P-element does appear to be less active in D. simulans $[32,45,55]$.

Another, simpler explanation for the difference in the speed of invasion is higher migration rates between $D$. simulans populations than for $D$. melanogaster as they experienced the $P$-element invasion. Such a scenario seems reasonable: Unlike D. melanogaster, D. simulans cannot over-winter in temperate regions, and instead reinvades the northern limits of its range annually. Sequence data correspondingly suggests a high rate of homogenization between $D$. simulans populations at nuclear loci [56-61]. Furthermore, for both species, migration might be largely human-aided, and the global movement of fruit has increased dramatically in the years since the spread of the $P$-element in D. melanogaster. That is, global imports and exports of fleshy fruits (measured in tons) grew about 4\% per year from 1961-2013 (the years tracked by the Food and Agriculture Organization of the United Nations Statistics Division). As a result, the average tonnage of fruit imports was 5-fold higher in the years measured during the spread of the $P$-element in D. simulans compared to D. melanogaster (for imports, 1961-1974: 12.5 vs. 2006-2014: 61.5 million tons/year; exports tracked imports closely). While these statistics capture only the movement of fruit between countries, the increase in movement seems likely to have boosted the global spread of the $P$-element in later years.

The $P$-element in $D$. simulans adds to a handful of documented cases of the spread of a horizontally transmitted element through a Drosophila species [33,62-65]. Several of the other cases include intracellular bacteria, which are capable of behaving selfishly, but in these cases also appear to be conferring a benefit to their hosts [64-67]. In contrast, the $P$-element appears to be spreading mainly via a selfish mechanism; though the occasional insertion may be beneficial $[22,68]$. In response, both $D$. simulans and D. melanogaster have quickly evolved to suppress the dysgenesis phenotype by the end of the invasion (present study, [20,38]), suggesting strong selection to ameliorate the the P-element's worst effects, and underscoring the evolutionary challenge posed by selfish elements.

\section{Materials and Methods}

\section{Fly strains}

We used D. simulans isofemale lines collected across multiple locations over 15 years (see S1 Table for details). Flies were reared on molasses-yeast-agar Drosophila medium at $18^{\circ} \mathrm{C}$ unless otherwise stated. The species identity of all lines was confirmed either by visual inspection of male genitalia or, when this was not possible, by amplification of PCR products that yield species-specific product lengths (using primer sequences for Argonaute2, FBgn0087035, kindly provided by D. Obbard: Mel_Sim_F 5'-CCCTAAACCGCAAGGATGGAG-3', D_sim_303_R 5'-GTCCACTTGTGCGCC ACATT-3', D_mel_394_R 5'-CCTTGCCTTGGGATATTATTAGGTT-3').

Unless otherwise stated, all lines were cured of Wolbachia infections before crosses were performed, in order to obtain sufficient offspring from crosses that normally show high levels of cytoplasmic incompatibility. (If known, the infection status of lines prior to curing is given in S1 Table.) To cure lines, we raised larvae on food supplemented with $0.05 \mathrm{mg} / \mathrm{ml}$ tetracycline-hydrochloride for two generations. After curing, flies were tested for Wolbachia using PCR, with primer sequences wsp81F ( $5^{\prime}$-TGGTCCAATAAGTGATGAAGAAAC- $\left.3^{\prime}\right)$ and wsp691R ( $5^{\prime}$-AAAAATTAAACGCTACTCCA- $3^{\prime}$ ), and a cycling program of $94^{\circ} \mathrm{C}$ for $5^{\prime}$ followed by 30 cycles of $94^{\circ} \mathrm{C} 30^{\prime \prime}, 55^{\circ} \mathrm{C}$ for $60^{\prime \prime}, 72^{\circ} \mathrm{C}$ for $2^{\prime}$, and ending with one cycle of $72^{\circ} \mathrm{C}$ for $10^{\prime}$, as in [69].

Hybrid dysgenesis assays. To assay for hybrid dysgenesis, we performed reciprocal crosses between lines using 5 virgin males and females from each line. Flies were left to lay eggs 
for a total of 9 days at $29^{\circ} \mathrm{C}$, transferred to a fresh vial every 3 days, and offspring reared at $29^{\circ} \mathrm{C}\left(28^{\circ} \mathrm{C}\right.$ is the lowest temperature at which dysgenesis is observed in D. melanogaster $[19,24])$. We dissected $30 \mathrm{~F} 1$ females from each cross, and assessed them for the presence or absence of two normal ovaries. We scored the number of females lacking one or both ovaries as dysgenic, following [24], and considered crosses showing a significant difference (at the 5\% level) to be dysgenic.

To classify strains as DI, DR, or DS, we reciprocally crossed each strain to at least one known DI and DS 'tester' strains, and roughly classified tested strains based on the results of the four crosses. Specifically, we defined strain as:

DI, if strain $\mathrm{x}$ tester DI, non-dysgenic; strain $\mathrm{x}$ tester DS, dysgenic

DS, if strain $\mathrm{x}$ tester DI, dysgenic; strain $\mathrm{x}$ tester DS, non-dysgenic

$\mathrm{DR}$, if strain $\mathrm{x}$ tester DI, non-dysgenic; strain $\mathrm{x}$ tester DS, non-dysgenic.

Some strains could not be unambiguously classified ( 5 borderline DI/DR types, and 6 borderline DR/DS types, and 3 that tested as both DI and DS). These strains were not used in the analysis; in all, 181 strains were unambiguously cytotyped.

Sterility assays. To perform the sterility assays shown in S4 Fig, we reciprocally crossed lines as described previously at $29^{\circ} \mathrm{C}$. For each line, individual virgin $\mathrm{F} 1$ females from both reciprocal crosses were mated to control virgin males (raised at $25^{\circ} \mathrm{C}$ ) at $25^{\circ} \mathrm{C}$. Flies were left to mate and lay eggs for a total of 7 days and then removed. The offspring from these crosses were collected and counted every 2 days. As fertility of F1 females from PxM crosses in D. melanogaster recovers with age [70], we checked the fertility of young (3-9 days old) and old (10-16 days) females separately. We compared the fertility of females from the 'dysgenic' and 'nondysgenic' direction of the crosses using a Mann-Whitney U test.

\section{Analysis of sequence data}

To find TEs expressed in the Florida lines, we used RNA-seq data form a pooled sample of lines from this population (accession number PRJEB7936 [31]). Paired end Illumina reads were mapped to a $D$. simulans reference genome produced from a Madagascar strain [44] and 179 Drosophila TE sequences (http://flybase.org/; [71,72]) using GSNAP ([73]; parameters: $-\mathrm{n}=1,-\mathrm{N}=1$ ).

We examined whole genome sequence from the Florida strains using paired-end Illumina reads (SRA PRJEB7936 [31], and PRJNA308281) collected from 12 barcoded individual F1 offspring of the M252 reference strain females (sequence data under SRX504933) crossed to males from Florida (2010) isofemale lines, and the paired end reads from the M252 reference strain [44]. We mapped these data to the D. simulans M252 reference genome using BWA-SW with the default parameters $[74,75]$ and following the mapping protocol described in [76]. We counted the number of insertions per individual sequenced in two ways: First, we used PoPoolationTE [74], with the requirement that each insertion be supported by at least 3 reads. Second, due to the fragmented nature of the $D$. simulans genome sequence, some insertion sites may not be recovered by PoPoolationTE, which relies on mapping reads to a reference. We therefore also estimated TE copy number per family by comparing average coverage of the TE sequence to average coverage of chromosome arm $2 \mathrm{~L}$.

\section{PCR assays}

Primers for TEs were designed based on the EMBL sequences (S4 Table, http://flybase.org/; [71,72]). The following PCR program was used: $94^{\circ} \mathrm{C}$ for $5^{\prime}$ followed by 30 cycles of $94^{\circ} \mathrm{C} 30^{\prime \prime}$, $55^{\circ} \mathrm{C}$ for $60^{\prime \prime}, 72^{\circ} \mathrm{C}$ for $1-3^{\prime}$ (depending on the expected length of the PCR product, 1 minute 
per kilobase); $72^{\circ} \mathrm{C}$ for $10^{\prime}$. PCR products were run on a $1 \%$ agarose gel to test for the presence or absence of a TE. To survey for the $P$-element, primers were designed for each exon of $P$-element (S4 Table).

The presence of full $P$-elements was assessed using the forward primer for exon 0 and the reverse primer for exon 3, and using a primer annealing to both ends of the $P$-element (in the TIR region; sequence kindly provided by L. Teysset). To ensure that PCR failures are due to the absence of $P$-element, DNA quality was checked with a positive control PCR for an essential gene, and D. melanogaster Harwich P was used as a positive control in all PCRs. Sanger sequencing (performed by LGC Genomics, http://www.lgcgroup.com/) of a subset of PCR products was used to confirm that the amplicons correspond to $P$-element sequence (Genbank accession numbers KU719478-KU719502); the sequence of additional sequencing primers is available upon request. We required a minimum of two independent DNA extractions to consistent results for each line.

To perform RT-PCR, RNA was isolated from Florida, Georgia, Harvard, Maryland and Madagascar lines using Peqlab TriFast RNA isolation protocol, and extractions checked on a $1 \%$ agarose gel following treatment with DNase. We then performed RT-PCR using QIAGEN OneStep RT-PCR Kit, using the following program to detect full-length $P$-element RNA (using the forward primer for exon 0 and the reverse primer for exon 3 ): $45^{\circ} \mathrm{C}$ for $30^{\prime}$ for reverse transcription, then $95 \mathrm{C}$ for $15^{\prime}$, followed by 30 cycles of $94 \mathrm{C}$ for $15^{\prime \prime}, 55 \mathrm{C}$ for $60^{\prime \prime}$ and $68 \mathrm{C}$ for 6 ; $68 \mathrm{C}$ for 10' to amplify cDNA. Using the same program, reverse transcription was also performed separately for both forward and reverse of the $P$-element (using either the forward primer for exon 0 or reverse for exon 3 ) to generate unidirectional cDNA for PCR.

\section{Statistical analyses}

All statistical analyses were performed using R [77]; https://www.r-project.org/). To estimate global trade in fruits, we downloaded data for total worldwide imports and exports of fruits and vegetables for the years 1961 to 2013 (measured in tons) from the FAOstat website (http:// faostat3.fao.org/download/T/TP/E). From these, data for nuts, vegetables, and processed foods were excluded, resulting in data for 29 categories of fleshy fruit and berries. Using the $n l s$ function in $\mathrm{R}$, we fit exponential growth models to these data of the form $y=a \exp (b x)$, where $y$ is the exports in tons, $x$ is the number of years since1961, and $a$ and $b$ are the estimated intercept and growth rate, respectively.

\section{Supporting Information}

S1 Fig. Dysgenic and non-dysgenic ovary dissections. Images showing non-dysgenic (A-F) and dysgenic ovaries (G-L). The brown bar on each image is a scale of $0.5 \mathrm{~mm}$. All ovaries were taken from F1 females from crosses between SGA35 \& M252 for each direction. Non-dysgenic ovaries, though they appear to vary in size and ovariole number (e.g. C vs. D), are much larger than dysgenic ovaries. In dysgenic ovaries, if present, they are usually rudimentary and only one or two ovarioles form properly (I-L). Dysgenic ovaries can also be asymmetric, as ovarioles will occasionally form on one side but not the other (I-K), as previously described in D. melanogaster [25]. Frequencies of total dysgenesis $(\mathrm{G} \& \mathrm{H})$ vs. asymmetrical dysgenesis (I-L) vs. rudimentary ovaries $(\mathrm{L})$ were not recorded during dissections, though total dysgenesis was seen in an estimated $70 \%$ of dysgenesis cases.

(TIF)

S2 Fig. Hybrid dysgenic crosses and controls. Bar plots show the fraction of dysgenic offspring for both directions of each set of reciprocal crosses. Bars are coloured red when a 
significant difference between each reciprocal cross is found (Fisher's Exact Test, $p<0.05$ ). A) Initial crosses between Georgia (2009) and Madagascar (2004) flies at $29^{\circ} \mathrm{C}$, with the fraction of dysgenic F1 females shown when the Georgia strain is paternal (positive direction) or maternal (negative direction). B) A subset of the crosses in panel A were repeated at $25^{\circ} \mathrm{C}$, a non-dysgenic temperature in D. melanogaster; repeated crosses are shown in the same position as in panel A. C) Fraction of dysgenic offspring from crosses within and between Georgia and Florida lines at $29^{\circ} \mathrm{C}$. The first strain named in the cross is the paternal strain in the positive direction and the maternal strain in the negative direction. D) Fraction of dysgenic offspring from crosses within and between Madagascar lines at $29^{\circ} \mathrm{C}$. The first strain named in the cross is the paternal strain in the positive direction and the maternal strain in the negative direction. E) Fraction of dysgenic offspring from crosses between Georgia and Madagascar lines at 29C after curing of Wolbachia with tetracycline hydrachloride for 2 generations. F) Fraction of dysgenic offspring from crosses between Georgia and Madagascar lines at 29C before curing. (PDF)

S3 Fig. Hybrid dysgenesis map. Map shows the approximate location where strains were collected; pie charts show the proportion of each population which were dysgenesis-inducing (red), dysgenesis-resistant (orange) and dysgenesis-susceptible (yellow). The area of the pie chart is proportional to the number of strains sampled. For raw data see: http://dx.doi.org/10. 5061/dryad.1rq8f. (PDF)

S4 Fig. Sterility assay. Plots A-C show the number of sterile F1 offspring produced from the dysgenic (red) and reciprocal (grey) crosses (from 35 total offspring), shown with $95 \%$ confidence interval. Crosses that show a significant difference in sterility between directions are marked with a star (Fisher's Exact Test $p<0.05$ ). Plots D-F show the number of offspring produced by the female offspring from both the dysgenic and reciprocal cross. Crosses that show a significant difference in the number of offspring for each direction are marked with a star (Wilcox Rank Sum Test $p<0.05)$. Female parents were dissected after crossing to confirm the presence of gonadal dysgenesis (mean of $50.6 \%$ of female parents were dysgenic, compared to a mean of $8.6 \%$ dysgenic female parents in the reciprocal cross, note this is a similar proportion to the proportion of sterile F1 females). A \& D. Females were mated after 3-9 days of aging. B \& E. Females were mated after 10-16 days of aging. C \& F. Combined results for all crosses and ages.

(PDF)

S5 Fig. TE coverage comparison. Average coverage for TEs in the sequenced Florida (2010) $\mathrm{x}$ Madagascar (2004) isofemale lines $v s$. coverage for TEs in M252 (2004). Here, coverage is defined as the average per base coverage of TE sequence, divided by the average per base coverage of chromosome $2 \mathrm{R}$ in the line. The Florida coverage is taken as the average coverage of 12 available lines (FL3, FL6, FL98, FL101, FL116, FL136, FL168, FL174, FL189, FL198, FL208 \& FL211). The Florida data (SRA:PRJEB7936, PRJNA308281) and Madagascar data (SRA:

SRX504933) was mapped to the $D$. simulans reference genome alongside Flybase TE sequences to identify differences in coverage for these TEs. Only one TE was found in Florida but not in Madagascar, P-element.

(PDF)

S6 Fig. TE copy number vs. strength of hybrid dysgenesis. Estimated copy number of TE (estimated via dividing the average coverage of the TE in a sample by the average coverage of chromosome 2R) $v s$. the fraction of dysgenic offspring observed in a cross (12 lines from Florida population [described in S5 Fig] crossed to M26 [in red] and M252 [in black]), with lines 
showing the fit of a binomial generalised linear model. A) The correlation between the copy number of P-element and the proportion of dysgenic offspring from the paternal Florida crosses ( $\mathrm{S} 2$ Table; $\mathrm{z}$ value $=6.49, p=8.49 \mathrm{e}-11$ ). The equivalent association between dysgenesis and P-element copy number for D. melanogaster is shown with clear points [16]. B) The correlation between the copy number of P-element and the proportion of dysgenic offspring from the maternal Florida crosses ( $\mathrm{S} 2 \mathrm{Table} ; \mathrm{z}$ value $=-1.128, p=0.259$ ). $\mathrm{C}$. The correlation between the copy number of Tom 1 and the proportion of dysgenic offspring from the paternal Florida crosses ( $\mathrm{S} 2$ Table; $\mathrm{z}$ value $=-0.551, p=0.581$ ). $\mathrm{D}$. The correlation between the copy number of Tom 1 and the proportion of dysgenic offspring from the maternal Florida crosses (S2 Table; $\mathrm{z}$ value $=0.135, p=0.893$ ).

(PDF)

S7 Fig. rtPCR for P-element in different line classifications. A) RT-PCR product from DI and DS lines. Group 1 consists of female flies from three DI lines roughly dissected into soma (S) and ovaries (G). The top band, at $2.6 \mathrm{~kb}$, corresponds to the expected size of the full-length P-element transcript after splicing. Group 2 consists of female flies from two DS lines with a positive control (D. melanogaster Harwich strain). B) PCR product from DI, DR \& DS lines following reverse transcription with either forward (1) or reverse (2) primers.

(PDF)

S1 Table. Fly strains used in this study. Table includes time and date of collection, and collector. The fraction of strains with P-element are also shown for each collection. (PDF)

S2 Table. Association between cytotypes of $D$. simulans strains and infection status for individual TE families. Number of reads mapping shows the number of reads which mapped to the TE in the expression data (SRA:PRJEB7936). The Z-value and P-value are from a binomial generalised linear model used to analyze the association between the TE copy number (found in the Florida sequence data, SRA:PRJEB7936, PRJNA308281) and the number of hybrid dysgenesis seen in a reciprocal crosses to an M line (see also S6 Fig); TEs causing the dysgenesis would be expected to show a significant relationship between dysgenesis in the cross where the Florida line is the male parent. Also shown is the number of DI strains and DS strains from which a TE could be amplified. Note that individual exons for the P-element could be amplified from all 39 DI strains; the results shown here are for amplification of the full- length P-element.

(PDF)

S3 Table. Generalized linear model fit to the data on PCR presence/absence data. The model used fit the year and region from which strains were collect (see S1 Table), plus an interaction term, to the presence or absence of P-element in that strain, and used a binomial error model. (i.e., the call in $\mathrm{R} \mathrm{glm}$ (formula $=$ cbind (present,absent $) \sim$ year ${ }^{*}$ region, family = "binomial")

(PDF)

S4 Table. Primers used for identifying the presence or absence of P-element. (PDF)

\section{Acknowledgments}

Thanks to Viola Nolte for assistance with PCR, performing the sequencing and for providing information for many of the isofemale lines. We are grateful for helpful discussion provided by 
R. Kofler, W. Miller, K. Senti, and C. Vogl, and to B. Charlesworth, G. Lee, and three anonymous reviewers whose comments greatly improved the manuscript. We are especially grateful to all the researchers who generously shared fly collections (S1 Table), as this work would not have been possible without them.

\section{Author Contributions}

Conceived and designed the experiments: TH AJB. Performed the experiments: TH. Analyzed the data: TH AJB. Contributed reagents/materials/analysis tools: CS. Wrote the paper: TH AJB.

\section{References}

1. McClintock B. Induction of instability at selected loci in maize. Genetics. 1953; 38: 579-599. PMID: 17247459

2. Orgel LE, Crick FHC. Selfish DNA: the ultimate parasite. Nature. 1980; 284: 604-607. PMID: 7366731

3. Doolittle WF, Sapienza C. Selfish genes, the phenotype paradigm and genome evolution. Nature. 1980; 284: 601-603. doi: 10.1038/284601a0 PMID: 6245369

4. Kidwell MG. Transposable elements and the evolution of genome size in eukaryotes. Genetica. 2002; 115: 49-63. doi: 10.1023/A:1016072014259 PMID: 12188048

5. Gregory TR. Synergy between sequence and size in large-scale genomics. Nat Rev Genet. 2005; 6 : 699-708. doi: 10.1038/nrg1674 PMID: 16151375

6. González J, Petrov DA. The adaptive role of transposable elements in the Drosophila genome. Gene. 2009; 448: 124-33. doi: 10.1016/j.gene.2009.06.008 PMID: 19555747

7. Biessmann H, Valgeirsdottir K, Lofsky A, Chin C, Ginther B, Levis RW, et al. HeT-A, a transposable element specifically involved in "healing" broken chromosome ends in Drosophila melanogaster. Mol Cell Biol. 1992; 12: 3910-3918. doi: 10.1128/MCB.12.9.3910.Updated PMID: 1324409

8. Ellison $\mathrm{CE}$, Bachtrog D. Dosage compensation via transposable element mediated rewiring of a regulatory network. Science. 2013; 342: 846-850. doi: 10.1126/science.1239552 PMID: 24233721

9. Lee YCG. The role of piRNA-mediated epigenetic silencing in the population dynamics of transposable elements in Drosophila melanogaster. PLOS Genet. 2015; 11: 1-24. doi: 10.1371/journal.pgen. 1005269

10. Pasyukova EG, Nuzhdin SV, Morozova TV, Mackay TFC. Accumulation of transposable elements in the genome of Drosophila melanogaster is associated with a decrease in fitness. J Hered. 2004; 95 : 284-90. doi: 10.1093/jhered/esh050 PMID: 15247307

11. Langley $\mathrm{CH}$, Montgomery $\mathrm{E}$, Hudson R, Kaplan N, Charlesworth B. On the role of unequal exchange in the containment of transposable element copy number. Genet Res. 1988; 52: 223-235. doi: 10.1017/ S0016672300027695 PMID: 2854088

12. Bellen HJ, Levis RW, He Y, Carlson JW, Evans-Holm M, Bae E, et al. The Drosophila gene disruption project: Progress using transposons with distinctive site specificities. Genetics. 2011; 188: 731-743. doi: 10.1534/genetics.111.126995 PMID: 21515576

13. Lohe AR, Moriyama EN, Lidholm DA, Hartl DL. Horizontal transmission, vertical inactivation, and stochastic loss of mariner-like transposable elements. Mol Biol Evol. 1995; 12: 62-72. doi: 10.1093/ oxfordjournals.molbev.a040191 PMID: 7877497

14. Hartl DL, Lozovskaya ER, Nurminsky DI, Lohe AR. What restricts the activity of mariner-like transposable elements? Trends Genet. 1997; 13: 197-201. doi: 10.1016/S0168-9525(97)01087-1 PMID: 9154003

15. Sánchez-Gracia A, Maside X, Charlesworth B. High rate of horizontal transfer of transposable elements in Drosophila. Trends Genet. 2005; 21: 200-203. doi: 10.1016/j.tig.2005.02.001 PMID: 15797612

16. Bingham PM, Kidwell MG, Rubin GM. The molecular basis of P-M hybrid dysgenesis: the role of the $P$ element, a P-strain-specific transposon family. Cell. 1982; 29: 995-1004. PMID: 6295641

17. Kidwell MG. Evolution of hybrid dysgenesis determinants in Drosophila melanogaster. Proc Natl Acad Sci U S A. 1983; 80: 1655-9. PMID: 6300863

18. Daniels SB, Peterson KR, Strausbaugh LD, Kidwell MG, Chovnick A. Evidence for horizontal transmission of the P transposable element between Drosophila species. Genetics. 1990; 124: 339-355. PMID: 2155157 
19. Kidwell JF, Kidwell MG, Sved JA. Hybrid dysgenesis in Drosophila melanogaster: A syndrome of aberrant traits including mutation, sterility, and male recombination. Genetics. 1977; 86: 813-833. PMID: 17248751

20. Anxolabéhère $D$, Kidwell MG, Periquet G. Molecular characteristics of diverse populations are consistent with the hypothesis of a recent invasion of Drosophila melanogaster by mobile P Elements. Mol Biol Evol. 1988; 5: 252-269. PMID: 2838720

21. Anxolabéhère $D, H u K$, Nouaud $D$, Periquet $G$. The distribution of the P-M system in Drosophila melanogaster strains from the People's Republic of China. Genet Sel Evol. 1990; 22: 175-188.

22. Engels WR. P elements in Drosophila. In: Saedley H, Gierl A, editors. Transposable Elements. Berlin: Springer-Verlag; 1996. pp. 103-123. Available: http://engels.genetics.wisc.edu/Pelements/Pt.html

23. Hiraizumi Y. Spontaneous recombination in Drosophila melanogaster males. Proc Natl Acad Sci U S A. 1971; 68: 268-70. PMID: 5277066

24. Engels WR, Preston CR. Hybrid Dysgenesis in Drosophila melanogaster: The Biology of Female and Male Sterility. Genetics. 1979; 92: 161-174. PMID: 115745

25. Engels WR. The P Family of Transposable Elements in Drosophila. Annu Rev Genet. 1983; 17: 315344. PMID: 6320712

26. Rubin GM, Kidwell MG, Bingham PM. The molecular basis of P-M hybrid dysgenesis: the nature of induced mutations. Cell. 1982; 29: 987-994. PMID: 6295640

27. Castro JP, Carareto CMA. Drosophila melanogaster $P$ transposable elements: mechanisms of transposition and regulation. Genetica. 2004; 121: 107-118. PMID: 15330110

28. Brennecke J, Malone CD, Aravin AA, Sachidanandam R, Stark A, Hannon GJ. An epigenetic role for maternally inherited piRNAs in transposon silencing. Science. 2008; 322: 1387-1392. doi: 10.1126/ science.1165171 PMID: 19039138

29. Rubin GM, Spradling AC. Genetic transformation of Drosophila with transposable element vectors. Science. 1982; 218: 348-353. doi: 10.1126/science.6289436 PMID: 6289436

30. Brookfield JFY. Apparent absence of transposable elements related to the P-elements of $D$. melanogaster in other species of Drosophila. Nature. 1984; 310: 330-332. PMID: 6087152

31. Kofler R, Hill T, Nolte V, Betancourt AJ, Schlötterer C. The recent invasion of natural Drosophila simulans populations by the P-element. Proc Natl Acad Sci. 2015; 112: 6659-6663. doi: 10.1073/pnas. 1500758112 PMID: 25964349

32. Montchamp-Moreau C. Dynamics of P-M hybrid dysgenesis in P-transformed lines of Drosophila simulans. Evolution. 1990; 44: 194-203.

33. Turelli M, Hoffman A. Rapid spread of an inherited incompatibility factor in California Drosophila. Nature. 353: 440-442. PMID: 1896086

34. Verspoor RL, Haddrill PR. Genetic diversity, population structure and Wolbachia infection status in a worldwide sample of Drosophila melanogaster and D. simulans populations. PloS One. $2011 ; 6$ : e26318. doi: 10.1371/journal.pone.0026318 PMID: 22022599

35. Kidwell MG. Hybrid dysgenesis in Drosophila melanogaster: the relationship between the P-M and I-R interaction systems. Genet Res. 1979; 33: 205-217. doi: 10.1017/S0016672300018358

36. Bregliano JC, Kidwell MG. Hybrid dysgenesis determinants. Mobile Genetic Elements. New York: J. A. Shapiro (Academic); 1983. pp. 363-410.

37. Anxolabéhère $D$, Nouaud $D$, Périquet $G$, Tchen $P$. P-element distribution in Eurasian populations of Drosophila melanogaster: A genetic and molecular analysis. Proc Natl Acad Sci U S A. 1985; 82 : 5418-22. PMID: 16593591

38. Black DM, Jackson MS, Kidwell MG, Dover GA. KP elements repress P-induced hybrid dysgenesis in Drosophila melanogaster. EMBO. 1987; 6: 4125-4135.

39. Malone CD, Hannon GJ. Molecular evolution of piRNA and transposon control pathways in Drosophila. Cold Spring Harb Symp Quant Biol. 2009; 74: 225-234. doi: 10.1101/sqb.2009.74.052 PMID: 20453205

40. Bucheton A, Paro R, Sang HM, Pelisson A, Finnegan DJ. The molecular basis of I-R hybrid Dysgenesis in Drosophila melanogaster: Identification, cloning, and properties of the I factor. Cell. 1984; 38: 153163. PMID: 6088060

41. Blackman RK, Grimaila R, Koehler MMD, Gelbart WM. Mobilization of hobo elements residing within the decapentaplegic gene complex: Suggestion of a new hybrid dysgenesis system in Drosophila melanogaster. Cell. 1987; 49: 497-505. PMID: 3032458

42. Pascua L, Periquet G. Distribution of hobo transposable elements in natural populations of Drosophila melanogaster. Mol Biol Evo. 1991; 8: 282-296. 
43. Evgen'ev MB, Zelentsova H, Shostak N, Kozitsina M, Barskyi V, Lankenau D, et al. Penelope, a new family of transposable elements and its possible role in hybrid dysgenesis in Drosophila virilis. Proc Natl Acad Sci U S A. 1997; 94: 196-201. PMID: 8990185

44. Palmieri N, Nolte V, Chen J, Schlötterer C. Genome assembly and annotation of a Drosophila simulans strain from Madagascar. Mol Ecol Resour. 2014; doi: 10.1111/1755-0998.12297

45. Daniels S, Chovnick A, Kidwell M. Hybrid dysgenesis in Drosophila simulans lines transformed with autonomous P elements. Genetics. 121: 281-291. PMID: 2731724

46. Marin L, Lehmann M, Nouaud D, Izaabel H, Anxolabehere D, Ronsseray S. P-Element repression in Drosophila melanogaster by a naturally occurring defective telomeric P copy. Genetics. 2000; 155: 1841-1854. PMID: 10924479

47. Brennecke J, Aravin AA, Stark A, Dus M, Kellis M, Sachidanandam R, et al. Discrete small RNA-generating loci as master regulators of transposon activity in Drosophila. Cell. 2007; 128: 1089-1103. doi: 10.1016/j.cell.2007.01.043 PMID: 17346786

48. Malone CD, Brennecke J, Dus M, Stark A, Mccombie WR, Sachidanandam R, et al. Specialized piRNA pathways act in germline and somatic tissues of the Drosophila ovary. Cell. 2009; 137: 522-535. doi: 10.1016/j.cell.2009.03.040 PMID: 19395010

49. Itoh M, Boussy IA. Full-size $P$ and $K P$ elements predominate in wild Drosophila melanogaster. Genes Genet Syst. 2002; 77: 259-267. doi: 10.1266/ggs.77.259 PMID: 12419898

50. Ogura K, Woodruff RC, Itoh M, Boussy IA. Long-term patter ns of genomic $P$ element content and $P-M$ characteristics of Drosophila melanogaster in eastern Australia. Genes Genet Syst. 2007; 82: 479-487. doi: 10.1266/ggs.82.479 PMID: 18270438

51. Sebnem Onder B, Erisöz Kasap O. P Element activity and molecular structure in Drosophila melanogaster populations from Firtina Valley, Turkey. J Insect Sci. 2014; 14: 16. doi: 10.1673/031.014.16 PMID: 25373163

52. Gloor GB, Nassif NA, Johnson-Schlitz DM, Preston CR, Engels WR. Targeted gene replacement in Drosophila via P element-induced gap repair. Science. 1991; 253: 1110-1117. PMID: 1653452

53. Matute DR, Ayroles JF. Hybridization occurs between Drosophila simulans and $D$. sechellia in the Seychelles archipelago. J Evol Biol. 2014; 27: 1057-68. doi: 10.1111/jeb.12391 PMID: 24773151

54. Lachaise D, David J, Lemeunier F, Tsacas L, Ashburner M. The Reproductive Relationships of Drosophila sechellia with $D$. mauritiana, $D$. simulans and $D$. melanogaster from Afrotropical Region. Evolution. 1986; 40: 262-271.

55. Kimura K, Kidwell MG. Differences in P element population dynamics between the sibling species Drosophila melanogaster and Drosophila simulans. Genet Res. 1994; 63: 27-38. PMID: 8206365

56. Bouletreau-Merle J, Fouillet $P$, Varaldi J. Divergent strategies in low temperature environment for the sibling species Drosophila melanogaster and $D$. simulans: overwintering in extension border areas of France and comparison with African populations. Evol Ecol. 2003; 17: 523-548.

57. Machado HE, Bergland AO, O'Brien KR, Behrman EL, Schmidt PS, Petrov DA. Comparative population genomics of latitudinal variation in $D$. simulans and $D$. melanogaster. Mol Ecol. 2015; n/a-n/a. doi: 10.1111/mec.13446

58. Ballard JW. Comparative genomics of mitochondrial DNA in Drosophila simulans. J Mol Evol. 2000; 51: 64-75. doi: 10.1007/s002390010067 PMID: 10903373

59. James a C, Ballard JW. Expression of cytoplasmic incompatibility in Drosophila simulans and its impact on infection frequencies and distribution of Wolbachia pipientis. Evol Int J Org Evol. 2000; 54: 1661-72. doi: 10.1111/j.0014-3820.2000.tb00710.x

60. Gravot E, Huet M, Veuille M. Effect of breeding structure on population genetic parameters in Drosophila. Genetics. 2004; 166: 779-788. PMID: 15020467

61. Hoffman A. Physiological climatic limits in Drosophila: patterns and implications. J Exp Biol. 2010; 213: 870-880. doi: 10.1242/jeb.037630 PMID: 20190112

62. Wilfert L, Jiggins FM. Flies on the move: an inherited virus mirrors Drosophila melanogaster's elusive ecology and demography. Mol Ecol. 2014; 23: 2093-2104. doi: 10.1111/mec.12709 PMID: 24597631

63. Riegler M, Sidhu M, Miller WJ, O'Neill SL. Evidence for a global Wolbachia replacement in Drosophila melanogaster. Curr Biol 2005; 15: 1428-33. doi: 10.1016/j.cub.2005.06.069 PMID: 16085497

64. Jaenike J, Unckless R, Cockburn SN, Boelio LM, Perlman SJ. Adaptation via symbiosis: Recent spread of a Drosophila defensive symbiont. Science. 2013;212: 212-215. doi: 10.1126/science.1188235

65. Kriesner P, Hoffmann AA, Lee SF, Turelli M, Weeks AR. Rapid sequential spread of two Wolbachia variants in Drosophila simulans. PLoS Pathog. 2013; 9: e1003607. doi: 10.1371/journal.ppat.1003607 PMID: 24068927 
66. Fry a J, Palmer MR, Rand DM. Variable fitness effects of Wolbachia infection in Drosophila melanogaster. Heredity. 2004; 93: 379-89. doi: 10.1038/sj.hdy.6800514 PMID: 15305172

67. Teixeira L, Ferreira A, Ashburner M. The bacterial symbiont Wolbachia induces resistance to RNA viral infections in Drosophila melanogaster. PLoS Biol. 2008; 6: e2. doi: 10.1371/journal.pbio.1000002

68. Schmidt JM, Good RT, Appleton B, Sherrard J, Raymant GC, Bogwitz MR, et al. Copy number variation and transposable elements feature in recent, ongoing adaptation at the Cyp6g1 Locus. PLoS Genet. 2010; 6: e1000998. doi: 10.1371/journal.pgen.1000998 PMID: 20585622

69. Braig HR, Zhou W, Dobson SL, Neill SLO. Cloning and characterization of a gene encoding the major surface protein of the bacterial endosymbiont Wolbachia pipientis. J Bacteriol. 1998; 180: 2373-2378. PMID: 9573188

70. Khurana JS, Wang J, Xu J, Koppetsch BS, Thomson TC, Nowosielska A, et al. Adaptation to P Element transposon invasion in Drosophila melanogaster. Cell. 2011; 147: 1551-1563. doi: 10.1016/j.cell.2011. 11.042 PMID: 22196730

71. Quesneville H, Bergman CM, Andrieu O, Autard D, Nouaud D, Ashburner M, et al. Combined evidence annotation of transposable elements in genome sequences. PLoS Comput Biol. 2005; 1: 0166-0175. doi: 10.1371/journal.pcbi.0010022

72. Kaminker JS, Bergman CM, Kronmiller B, Carlson J, Svirskas R, Patel S, et al. The transposable elements of the Drosophila melanogaster euchromatin: a genomics perspective. Genome Biol. 2002; 3: RESEARCH0084. doi: 10.1186/gb-2002-3-12-research0084 PMID: 12537573

73. Wu TD, Nacu S. Fast and SNP-tolerant detection of complex variants and splicing in short reads. Bioinforma Oxf Engl. 2010; 26: 873-881. doi: 10.1093/bioinformatics/btq057

74. Li H, Durbin R. Fast and accurate short read alignment with Burrows-Wheeler transform. Bioinforma Oxf Engl. 2009; 25: 1754-60. doi: 10.1093/bioinformatics/btp324

75. Li H, Handsaker B, Wysoker A, Fennell T, Ruan J, Homer N, et al. The Sequence Alignment/Map format and SAMtools. Bioinforma Oxf Engl. 2009; 25: 2078-9. doi: 10.1093/bioinformatics/btp352

76. Kofler R, Betancourt AJ, Schlötterer C. Sequencing of pooled DNA Samples (Pool-Seq) uncovers complex dynamics of transposable element insertions in Drosophila melanogaster. PloS Genet. 2012; 8: 116. doi: 10.1371/journal.pgen.1002487

77. Team RC. R: A Language and Environment for Statistical Computing [Internet]. Vienna, Austria: R Foundation for Statistical Computing; 2013. Available: http://www.r-project.org 